\title{
Graviton multipoint amplitudes for higher-derivative gravity in anti-de Sitter space
}

\author{
M. M. W. Shawa ${ }^{1, *}$ and A. J. M. Medved ${ }^{1,2, \uparrow}$ \\ ${ }^{1}$ Department of Physics \& Electronics, Rhodes University, Grahamstown 6140, South Africa \\ ${ }^{2}$ National Institute for Theoretical Physics (NITheP), Western Cape 7602, South Africa
}

(Received 9 September 2017; published 3 April 2018)

\begin{abstract}
We calculate graviton multipoint amplitudes in an anti-de Sitter black brane background for higherderivative gravity of arbitrary order in numbers of derivatives. The calculations are performed using tensor graviton modes in a particular regime of comparatively high energies and large scattering angles. The regime simplifies the calculations but, at the same time, is well suited for translating these results into the language of the dually related gauge theory. After considering theories whose Lagrangians consist of contractions of up to four Riemann tensors, we generalize to even higher-derivative theories by constructing a "basis" for the relevant scattering amplitudes. This construction enables one to find the basic form of the $n$-point amplitude for arbitrary $n$ and any number of derivatives. Additionally, using the fourpoint amplitudes for theories whose Lagrangians carry contractions of either three or four Riemann tensors, we reexpress the scattering properties in terms of the Mandelstam variables.
\end{abstract}

DOI: 10.1103/PhysRevD.97.086002

\section{INTRODUCTION}

The gauge-gravity duality enables one to describe a $d$-dimensional gauge theory in terms of a $(d+1)$ dimensional gravitational theory [1]. Importantly, the duality relates a strongly coupled field theory to a weakly coupled theory of gravity. Since strongly coupled gauge theories are not very well understood, the duality provides a means for making analytical statements about them. One application of this framework [2] is the correspondence between stress-energy tensor correlation functions in the relevant gauge theory and graviton scattering amplitudes in its gravitational dual [3].

In the earliest investigations into the duality-which mostly focused on five-dimensional anti-de Sitter (AdS) space and four-dimensional super Yang-Mills theory-the rank of the gauge theory $N$ is taken to infinity, which then corresponds to Einstein's theory of gravity [4]. (On the other hand, the 't Hooft coupling $\lambda=g_{s}^{2} N$, where $g_{s}$ is the string coupling, is regarded as large but finite in the standard limit.) With deviations to large but finite values of $N$, the gravitational dual can be expected to include higher-derivative corrections in addition to Einstein's (two-derivative)

\footnotetext{
*markshawa@aims.ac.za

j.medved@ru.ac.za
}

Published by the American Physical Society under the terms of the Creative Commons Attribution 4.0 International license. Further distribution of this work must maintain attribution to the author(s) and the published article's title, journal citation, and DOI. Funded by SCOAP.
Lagrangian and two-derivative field equation [5]. If the interest is only in gauge-invariant quantities (such as scattering amplitudes), then one can limit considerations to the multiderivative terms in the Lagrangian which are strictly composed of contractions between "proper" four-index Riemann tensors (i.e., two-index tensors and scalars are excluded). This simplification was recently discussed in [6] and can be shown through a gauge transformation of the graviton that involves the Ricci scalar and tensor $[7,8]$.

A brief note on conventions: A theory of gravity whose Lagrangian consists solely of terms containing contractions of $k$ Riemann tensors will be called Riem ${ }^{k}$ gravity. The field equation for such a theory could have up to $2 k$ derivatives in any given term, but its highest-order term could still have less than $2 k$ derivatives (with Gauss-Bonnet gravity serving as a case in point; see below).

In this paper, we calculate graviton scattering amplitudes for higher-derivative theories of gravity in an AdS black brane background, that is, a black hole with a flat horizon living in an asymptotically AdS spacetime. Our approach is similar to that of [9], where the focus is on Einstein (Riem ${ }^{1}$ ) and Gauss-Bonnet $\left(\mathrm{Riem}^{2}\right)$ gravity. However, those scattering amplitudes could be substantially simplified by enforcing on-shell conditions. In the case of Einstein's gravity, the field equation can be used to eliminate amplitudes with two derivatives acting on a single graviton. Meanwhile, any formulation of Riem ${ }^{2}$ gravity can always be gauge transformed to a Gauss-Bonnet theory by using the "inverse" of the aforementioned transformation. As any Lovelock extension of Einstein's theory [10], GaussBonnet gravity has a field equation that has at most two 
derivatives per term irrespective of how many Riemann tensors are carried in the Lagrangian. Thus, the very same logic and simplification applies in this case as well. (See the appendix in [9] for further details.) In the current work, we do not have the luxury of restricting to Lovelock theories or theories that are related to Lovelock via a gauge transformation, ${ }^{1}$ meaning that the previous simplification can no longer be relied upon. It follows that terms with two derivatives acting on a graviton must now be included in the calculation of amplitudes.

Starting with $\mathrm{Riem}^{3}$ and Riem ${ }^{4}$ theories in Sec. II, we utilize what could be called "basis amplitudes" to construct the $n$-point amplitudes for arbitrarily large $n$. The generalization of these basis amplitudes mitigates the task of finding multipoint amplitudes for theories with an arbitrary number of Riemann tensors (see Sec. III). Finally, using 4-point amplitudes in Sec. IV, we reexpress the scattering properties of the Riem ${ }^{3}$ and Riem ${ }^{4}$ theories in terms of the Mandelstam variables. (Section V contains a brief conclusion.)

Our intention is, in a later paper, to map the current results to stress-tensor correlation functions in the dual gauge theory. The motivation for such a treatment is to learn about the gravitational dual to the gauge theory describing the quark-gluon plasma and other strongly coupled gauge theories [11]. These findings could conceivably be tested thanks to the high-energy hadron-collider laboratories at Brookhaven and CERN. For a detailed discussion on the physical significance of these correlation functions and the viability of the high-momentum regime in this context, the reader can consult [12].

Because our long-term goal is to apply the gauge-gravity duality for the purpose of making experimentally viable predictions (as in [13]), we are mainly interested in the boundary limit of the amplitudes (where the gauge theory can be regarded as "residing") and, moreover, only those contributions that would survive holographic renormalization [14] and could be discernible in gauge-theory correlation functions. With this in mind, our calculations are limited to a special kinematic region that was referred to as the "highmomentum regime" in [9]. As discussed later, this regime is particularly well suited for discriminating the higherderivative contributions in the graviton scattering amplitudes and then, ultimately, in the dual correlation functions.

\section{A. Summary of formalism}

Much of the groundwork for the following analysis has already been laid out in $[9,13]$. Here, we will summarize some of the key elements that are necessary for the task at hand.

The AdS black brane background in a five-dimensional spacetime $^{2}$ has a metric of the form

\footnotetext{
${ }^{1}$ This follows from any Riem $^{k}$ Lovelock extension vanishing identically in $2 k-1$ or fewer dimensions [10].

${ }^{2}$ Generalizations to other dimensionalities are straightforward.
}

$d s^{2}=-f(r) d t^{2}+\frac{1}{g(r)} d r^{2}+\frac{r^{2}}{L^{2}}\left(d x^{2}+d y^{2}+d z^{2}\right)$,

where the radial coordinate ranges from the black brane horizon at $r=r_{h}$ to the AdS boundary limit of $r \rightarrow \infty$, and the parameter $L$ is the AdS radius of curvature. As for the functions $f(r)$ and $g(r)$, for an AdS black brane spacetime, these would be $f(r)=g(r)=\frac{r^{2}}{L^{2}}\left(1-\frac{r_{h}^{4}}{r^{4}}\right)$. However, in general, $f(r) \neq g(r)$, and these are chosen so that the metric is asymptotically pure AdS, meaning that $f(r)$ and $g(r)$ necessarily have to satisfy the four conditions $\lim _{r \rightarrow r_{h}} f(r)=0, \lim _{r \rightarrow r_{h}} g(r)=0, \lim _{r \rightarrow \infty} \frac{f(r)}{r^{2}}=\frac{1}{L^{2}}$, and $\lim _{r \rightarrow \infty} \frac{g(r)}{r^{2}}=\frac{1}{L^{2}}$.

Metric fluctuations are represented by $\bar{g}_{\mu \nu}=g_{\mu \nu}+h_{\mu \nu}$, where $g_{\mu \nu}$ is the AdS background metric of Eq. (1) and $h_{\mu \nu}$ is a small perturbation of the background metric (i.e., a graviton). In this analysis, the gravitons will propagate along the $z$ coordinate and so

$$
h_{a b}=\phi(r) e^{i(\omega t-k z)},
$$

where $\phi(r)$ is some well-behaved function with incomingwave boundary conditions at the horizon of the form [15]

$$
\phi(r)=f(r)^{-\frac{i \omega}{2 \pi T}} \psi(r),
$$

where $\psi(r)$ is finite and continuous near $r_{h}, \omega$ is the mode frequency, and $T$ is the Hawking temperature of the black brane, $T=\frac{r_{h}}{4 \pi L^{2}}$. Near the AdS boundary, $\phi(r)$ must be finite and continuous as well as depend on some negative power of $r$.

We assume a string-theory based gravitational theory with weak coupling, $g_{s} \ll 1$, and with a small string tension, $\epsilon=\frac{\ell_{s}^{2}}{L^{2}} \ll 1\left(\ell_{s}\right.$ is the string length). The former condition is needed to comply with the 't Hooft limit of large $N$ and large but relatively smaller $\lambda$. The latter condition allows us to consider a perturbative expansion of the Lagrangian in terms of $\epsilon$. The expansion can be expressed schematically as

$$
\begin{aligned}
\mathcal{L}= & \sqrt{-g} \frac{R}{16 \pi G_{5}}\left(1+\epsilon L^{2} R+\epsilon^{2} L^{4} R^{2}+\cdots\right. \\
& \left.+\epsilon^{k} L^{2 k} R^{k}+\cdots\right),
\end{aligned}
$$

where we have suppressed indices (all terms are contractions of 4-index Riemann tensors as previously discussed) and $G_{5}$ is Newton's constant in five dimensions. For the sake of brevity, the Lagrangian can also be written as

$$
\mathcal{L}=\sum_{k=1} L_{k}
$$

where $L_{k} \sim R^{k}$ is meant to describe a contraction of $k$ Riemann tensors. So that, when referring to (e.g.) $\mathrm{Riem}^{3}$ gravity, we really mean the Lagrangian $L_{3}=\frac{\sqrt{-g} R}{16 \pi G_{5}}\left[\epsilon^{2} L^{4} R^{2}\right]$. 
Gravitational scattering amplitudes must be holographically renormalized if we are to make a connection with the gauge-gravity duality. As shown in [9], this process eliminates the need to consider the radial derivatives of gravitons as these would lead to divergences in the amplitude even after renormalization. Such terms would then be discarded in the gauge theory after the standard techniques of holographic renormalization have been applied [14]. Hence, for the current work, only the $t$ and $z$ derivatives of gravitons are considered.

Let us now clarify what is meant by the "high-momentum regime." Our motivation is the idealized case of a kinematic region that is potentially accessible via experiment on the dual gauge theory ${ }^{3}$ and that allows one to distinguish between the contributions of the different $L_{k}$ 's in Eq. (5). The basic idea is that larger values of momenta can compensate for larger powers of $\epsilon$ as one probes theories that are higher order in numbers of derivatives. This is accomplished by insisting that a contribution to amplitudes of order $\epsilon^{q}$ always includes a total of $2 q+2$ derivatives acting on gravitons. Contributions having fewer derivatives are to be discarded. So that, if $L^{2} \epsilon \omega^{2}$ is not too small a number, the surviving contributions should be prominent in the scattering profiles (and ultimately in the gauge-theory correlators).

Let us explain further what classifies a value of momentum as "high" in this context. First, background derivatives go as $\nabla_{r} \nabla_{r} \sim \frac{1}{L^{2}}$, while graviton momenta go as $\nabla_{t} \nabla_{t} \sim \omega^{2}$ and $\nabla_{z} \nabla_{z} \sim k^{2}$; therefore, one requirement is that $\omega^{2}$, $k^{2} \gg \frac{1}{L^{2}}$. Furthermore, to apply the tools of the gaugegravity duality, the hydrodynamic regime must necessarily be in effect. ${ }^{4}$ This means that $\omega \ll T$ and, consequently,

$$
1 \ll L \omega \ll T L .
$$

This range is indeed viable because, according to the duality, $T L \sim \frac{r_{h}}{L} \gg 1$ [3]. One then needs only to hope that $\epsilon(\omega L)^{2}$ is sufficiently large, for the purpose of experimental detection (as always, on the gaugetheory side).

We also work in the radial gauge, which means that $h_{r a}=0$ for any $a$. This gauge divides the gravitional perturbations into three distinct sectors: scalar, vector, and tensor [17]. However, scalar modes do not contribute to scattering amplitudes in the high-momentum regime. This is because scalars need to be sourced and sources can be expected, on general grounds, to introduce an additional factor of $\epsilon$. Meanwhile, vector modes are analogous to the

\footnotetext{
${ }^{3}$ The viability of experimental accessibility hinges on having $\epsilon$ small but not too small. Essentially $\epsilon \sim \mathcal{O}\left(10^{-1}\right)$, although this number depends very much on parameters of the particular experiment. See [12] for further details.

${ }^{4}$ For another study on brane hydrodynamics in the context of higher-derivative gravity, see (e.g.) [16].
}

electromagnetic potential and, as such, can either be gauged away or require a source when appearing in gauge-invariant combinations. Hence, these modes can also be discounted. Other fields can only couple to the gravitons through a stress tensor, which will invoke additional powers of $\epsilon$. This leaves only the tensor modes $h_{x y}$ as relevant in the high-momentum regime.

Given that the number of derivatives acting on gravitons has been maximized, the only other type of tensor-mode amplitude requiring suppression is one that includes the on-shell constraint $\square h_{x y}=0+\mathcal{O}(\epsilon)$, since this adds an extra factor of $\epsilon$. Note though that an expression such as $g^{a b} g^{c d} \nabla_{a} \nabla_{c} h_{x y}$ would survive (with the free indices suitably contracted). We should also add that, to maintain general covariance, tensor modes must come in pairs (see below). And so, with the restriction to tensor modes, any nontrivial scattering amplitude will necessarily be even.

Finally, we will perturbatively expand the metric determinant and contravariant metrics by adopting the conventions of [18]. To briefly review, a perturbed covariant metric goes as

$$
\bar{g}_{\mu \nu}=g_{\mu \nu}+h_{\mu \nu}
$$

which is to be viewed as an exact statement up to any order in $h$. This expansion has a contravariant counterpart of the form

$$
\bar{g}^{\mu \nu}=g^{\mu \nu}-h^{\mu \nu}+h_{\rho}^{\mu} h^{\rho \nu}+\mathcal{O}\left(h^{3}\right),
$$

and the metric determinant is then

$\sqrt{-\bar{g}}=\sqrt{-g}\left[1+\frac{1}{2} h_{\mu}^{\mu}-\frac{1}{4} h_{\rho}^{\mu} h_{\mu}^{\rho}+\frac{1}{8}\left(h_{\mu}^{\mu}\right)^{2}+\mathcal{O}\left(h^{3}\right)\right]$.

Since our interest is in the tensor modes $h_{x y}$, only expressions with an even number of gravitons will survive in Eqs. (8) and (9), and undifferentiated gravitons will have to come from either

$$
\bar{g}^{x x}=g^{x x}+h_{y}^{x} h^{y x}+\left(h_{y}^{x} h^{y x}\right)^{2}+\cdots+\left(h_{y}^{x} h^{y x}\right)^{p}
$$

or

$\sqrt{-\bar{g}}=\sqrt{-g}\left[1-\frac{1}{2} h_{y}^{x} h_{x}^{y}-\frac{1}{2^{2} 2 !}\left(h_{y}^{x} h_{x}^{y}\right)^{2}+\cdots+\Theta(p)\left(h_{y}^{x} h_{x}^{y}\right)^{p}\right]$,

where

$$
\Theta(p)=-\frac{\Gamma\left[p-\frac{1}{2}\right]}{2 \sqrt{\pi} p !}, \quad \text { for } p \in \mathbb{Z} .
$$

As for the differentiated gravitons, these will, of course, come from expansions of the Riemann tensors (see below). 


\section{MULTIPOINT AMPLITUDES}

\section{A. Basis multipoint amplitudes}

Using the standard definition of Riemann tensors and Eq. (7), we start here by introducing some shorthand notation for perturbations of the Riemann tensor while considering only the highest momentum terms,

$$
\begin{gathered}
\delta^{(1)} \mathcal{R}_{a b c d}=\nabla_{b} \Gamma_{d a c}(h)-\nabla_{c} \Gamma_{d a b}(h), \\
\delta^{(2)} \mathcal{R}_{a b c d}=g^{e f}\left[\Gamma_{e a c}(h) \Gamma_{f b d}(h)-\Gamma_{e a d}(h) \Gamma_{f b c}(h)\right],
\end{gathered}
$$

where

$$
\Gamma_{a b c}(h)=\frac{1}{2}\left(\nabla_{b} h_{a c}+\nabla_{c} h_{a b}-\nabla_{a} h_{b c}\right) .
$$

Given that the high-momentum regime is in effect (so that the background contributions from any Riemann tensor can be ignored), then each Riemann tensor effectively contributes

$R_{a b c d} \rightarrow \mathcal{R}_{a b c d} \equiv \delta^{(1)} \mathcal{R}_{a b c d}+\delta^{(2)} \mathcal{R}_{a b c d} \sim \nabla \nabla h+\nabla h \nabla h$.

Note that additional derivatives on a graviton would only be possible through an integration by parts, which is inconsequential to on-shell amplitudes. Also, as any term in a strictly covariant Riemann tensor consists of at most two differentiated covariant metrics and zero differentiated contravariant metrics, there are no higher-order corrections to the above expressions [cf. Eq. (7) and the comment that follows it]. Nonetheless, higher-order terms in the complete amplitudes will come about from the expansions in Eqs. (8)-(11).

Recalling that gravitons can only be differentiated with respect to $t$ and $z$, one can observe that any perturbed Riemann tensor must have a specific arrangement of indices. Up to symmetries, ${ }^{5}$ these would be $\delta^{(1)} \mathcal{R}_{a x b y}$ for $a, b=$ $\{t, z\}$ and $\delta^{(2)} \mathcal{R}_{c x d x}$ for $c, d=\{t, z, y\}$, where $x$ and $y$ are interchangeable.

With the high-momentum regime in mind, we will define basis amplitudes as multipoint amplitudes for which all of the included gravitons are differentiated. Each such basis amplitude then represents a different combination of $\delta^{(1)} \mathcal{R}$ 's and $\delta^{(2)} \mathcal{R}$ 's. Furthermore, in constructing a general $2 n$-point amplitude, we will use the notation $\left\langle h^{2 n}\right\rangle_{2 p}$ to indicate a $2 n$-point amplitude constructed from a $2 p$-point basis amplitude $(n \geq p)$. For instance, the basis amplitudes themselves are denoted by $\left\langle h^{2 p}\right\rangle_{2 p}$.

\footnotetext{
${ }^{5}$ In particular, $\mathcal{R}_{a b c d}=\mathcal{R}_{c d a b}$ and $\mathcal{R}_{a b c d}=-\mathcal{R}_{a b d c}$.
}

\section{B. Multipoint amplitudes from Riem $^{3}\left(\epsilon^{2}\right.$-order $)$ gravity}

To begin, Riem ${ }^{3}$ gravity can be defined, up to gauge transformations, as

$L_{3}=\sqrt{-g}\left[\alpha R_{a b c d} R^{a b}{ }_{m n} R^{m n c d}+\beta R_{a b c d} R_{m n}{ }^{a d} R^{m n c b}\right]$,

where $\frac{\epsilon^{2} L^{4}}{16 \pi G_{5}}$ has now been absorbed into the modeldependent constants $\alpha$ and $\beta$.

In this theory, there two types of basis amplitudes, ${ }^{6}$ the 4- and 6-point amplitudes. The basis 4-point amplitude has six derivatives and four gravitons; schematically it can written in terms of the previously introduced shorthand [cf. Eqs. (13) and (14)],

$$
\left\langle h^{4}\right\rangle_{4} \sim 3(\alpha+\beta) \delta^{(1)} \mathcal{R} \delta^{(1)} \mathcal{R} \delta^{(2)} \mathcal{R},
$$

where the tensor indices have been suppressed and the " 3 " counts the number of ways of choosing one of the tensors to carry two gravitons.

Similarly, the 6-point amplitude with six derivatives and six gravitons takes the schematic form

$$
\left\langle h^{6}\right\rangle_{6} \sim(\alpha+\beta) \delta^{(2)} \mathcal{R} \delta^{(2)} \mathcal{R} \delta^{(2)} \mathcal{R} .
$$

Now more explicitly, the 4-point amplitude of Eq. (18) can be expressed as

$$
\begin{aligned}
\left\langle h^{4}\right\rangle_{4}= & 3(\alpha+\beta) 2^{3}\left[\mathcal{R}_{t x t y} \mathcal{R}^{t x t y} \mathcal{R}^{t x}{ }_{t x}+\mathcal{R}_{t x z y} \mathcal{R}^{t x z y} \mathcal{R}^{t x}{ }_{t x}\right. \\
& \left.+\mathcal{R}_{t x z y} \mathcal{R}^{z x z y} \mathcal{R}^{t x}{ }_{z x}+\{t \leftrightarrow z\}\right]+\{x \leftrightarrow y\},
\end{aligned}
$$

where $\{a \leftrightarrow b\}$ is shorthand for the interchange of $a$ and $b$ in the preceding expression and the $2^{3}$ accounts for the symmetries of the Riemann tensors. Even more explicitly, in terms of gravitons (and with the help of the equations in Sec. II A),

$$
\begin{aligned}
\left\langle h^{4}\right\rangle_{4}= & -6(\alpha+\beta) \sqrt{-g}\left(g^{x x} g^{y y}\right)^{2} \\
& \times\left[\left(\omega_{1} g^{t t} \omega_{2}+k_{1} g^{z z} k_{2}\right)\left(\omega_{1} g^{t t} \omega_{3}+k_{1} g^{z z} k_{3}\right)\right. \\
& \left.\times\left(\omega_{2} g^{t t} \omega_{4}+k_{2} g^{z z} k_{4}\right)\right] \\
& \times h_{x y}^{(1)} h_{x y}^{(2)} h_{x y}^{(3)} h_{x y}^{(4)},
\end{aligned}
$$

where each factor of $\left(\omega_{i} g^{t t} \omega_{j}+k_{i} g^{z z} k_{j}\right)$ is a product of the momenta for gravitons $h_{x y}^{(i)}$ and $h_{x y}^{(j)}$, and the symmetrization of the gravitons is always implied.

Using similar reasoning, one finds that the basis 6-point amplitude translates into

\footnotetext{
${ }^{6}$ Here, we will be disregarding 2-point amplitudes because they are not really of interest from the viewpoint of someone discriminating between different theories.
} 


$$
\begin{aligned}
\left\langle h^{6}\right\rangle_{6}= & -\frac{3}{2}(\alpha+\beta) \sqrt{-g}\left(g^{x x} g^{y y}\right)^{3}\left[\left(\omega_{1} g^{t t} \omega_{2}+k_{1} g^{z z} k_{2}\right)\left(\omega_{3} g^{t t} \omega_{4}+k_{3} g^{z z} k_{4}\right)\left(\omega_{5} g^{t t} \omega_{6}+k_{5} g^{z z} k_{6}\right)\right] \\
& \times h_{x y}^{(1)} h_{x y}^{(2)} h_{x y}^{(3)} h_{x y}^{(4)} h_{x y}^{(5)} h_{x y}^{(6)} .
\end{aligned}
$$

The utility of the basis amplitudes in Eqs. (21) and (22) is that one can use these to construct $2 n$-point amplitudes by drawing out additional pairs of gravitons from the metric determinant and the contravariant metrics $g^{x x}$ and $g^{y y}$. Let us begin with Eq. (21) and suppose that $p$ pairs are drawn from the metric determinant and $n-2-p$ pairs from the four contravariant metrics. Recalling that the number of ways of drawing $q$ identical objects from $m$ distinct boxes is $\left(\begin{array}{c}q+m-1 \\ m-1\end{array}\right)$, we then have that, for any $n \geq 2$,

$$
\begin{aligned}
\left\langle h^{2 n}\right\rangle_{4}= & -6(\alpha+\beta)\left(\begin{array}{c}
2 n \\
4
\end{array}\right) \sum_{p=0}^{n-2}\left(\begin{array}{c}
n+1-p \\
3
\end{array}\right) \Theta(p) \sqrt{-g}\left(g^{x x} g^{y y}\right)^{2} \\
& \times\left[\left(\omega_{1} g^{t t} \omega_{2}+k_{1} g^{z z} k_{2}\right)\left(\omega_{1} g^{t t} \omega_{3}+k_{1} g^{z z} k_{3}\right)\left(\omega_{2} g^{t t} \omega_{4}+k_{2} g^{z z} k_{4}\right)\right] \\
& \times h_{x y}^{(1)} h_{x y}^{(2)} h_{x y}^{(3)} h_{x y}^{(4)} \prod_{j=3}^{n}\left[\left(h_{y}^{x}\right)^{(2 j-1)}\left(h_{x}^{y}\right)^{(2 j)}\right]
\end{aligned}
$$

where the combinatorial factor before the summation represents the number of ways of choosing the four differentiated gravitons from the total of $2 n$ and the summation itself accounts for all possible ways of drawing gravitons from the contravariant metrics and metric determinant.

In similar fashion, $2 n$-point amplitudes can be constructed from the basis 6-point amplitude of Eq. (22) for any $n \geq 3$. The result of this is

$$
\begin{aligned}
\left\langle h^{2 n}\right\rangle_{6}= & -\frac{3}{2}(\alpha+\beta)\left(\begin{array}{c}
2 n \\
6
\end{array}\right) \sum_{q=0}^{n-3}\left(\begin{array}{c}
n-q+2 \\
5
\end{array}\right) \Theta(q) \sqrt{-g}\left(g^{x x} g^{y y}\right)^{3} \\
& \times\left[\left(\omega_{1} g^{t t} \omega_{2}+k_{1} g^{z z} k_{2}\right)\left(\omega_{3} g^{t t} \omega_{4}+k_{3} g^{z z} k_{4}\right)\left(\omega_{5} g^{t t} \omega_{6}+k_{5} g^{z z} k_{6}\right)\right] \\
& \times h_{x y}^{(1)} h_{x y}^{(2)} h_{x y}^{(3)} h_{x y}^{(4)} h_{x y}^{(5)} h_{x y}^{(6)} \prod_{k=4}^{n}\left[\left(h_{y}^{x}\right)^{(2 k-1)}\left(h_{x}^{y}\right)^{(2 k)}\right] .
\end{aligned}
$$

As Eqs. (23) and (24) now make clear, the 4- and 6-point amplitudes of Eqs. (21) and (22) form the basis for the $2 n$-point amplitudes of Riem ${ }^{3}$ gravity in the high-momentum regime. The complete $2 n$-point amplitude for Riem ${ }^{3}$ gravity is then given by the linear combination

$$
\left\langle h^{2 n}\right\rangle_{\operatorname{Riem}^{3}}=\left\langle h^{2 n}\right\rangle_{4}+\left\langle h^{2 n}\right\rangle_{6}
$$

\section{Multipoint amplitudes from $\mathrm{Riem}^{4}\left(\epsilon^{3}\right.$-order) gravity}

First consider that Riem ${ }^{4}$ gravity is expressible, up to gauge transformations, as

$$
\begin{aligned}
L_{4}= & {\left[\alpha R_{a b c d} R^{a b m n} R_{m n}{ }^{p q} R_{p q}{ }^{c d}+\beta R_{a b c d} R^{a b}{ }_{q p} R^{m n d q} R_{m n}{ }^{c p}+\gamma R_{a b c d} R^{p d}{ }_{m n} R^{a b c q} R_{p q}{ }^{m n}\right.} \\
& \left.+\mu R_{a b c d} R_{m n p q} R^{a n c q} R^{m b p d}+\nu R_{a b c d} R^{n a}{ }_{p q} R^{m b p d} R_{m n}{ }^{q c}+\rho R^{a b c d} R_{a b c d} R^{m n p q} R_{m n p q}\right] .
\end{aligned}
$$

Here, as before, $\frac{\epsilon^{3} L^{6}}{16 \pi G_{5}}$ has been absorbed into the model-dependent constants.

With the very same reasoning as in the previous section, we can call on Eq. (26) to construct three types of basis amplitudes; the 4-, 6-, and 8-point amplitudes. In terms of the Riemann tensor in Sec. II A, these can be schematically written as

$$
\begin{gathered}
\left\langle h^{4}\right\rangle_{4} \sim(\alpha+\beta+\gamma+\mu+\nu+\rho) \delta^{(1)} \mathcal{R} \delta^{(1)} \mathcal{R} \delta^{(1)} \mathcal{R} \delta^{(1)} \mathcal{R}, \\
\left\langle h^{6}\right\rangle_{6} \sim 6\left(\alpha+\beta+\gamma+\mu+\nu+\frac{1}{3} \rho\right) \delta^{(1)} \mathcal{R} \delta^{(1)} \mathcal{R} \delta^{(2)} \mathcal{R} \delta^{(2)} \mathcal{R},
\end{gathered}
$$




$$
\left\langle h^{8}\right\rangle_{8} \sim(\alpha+\beta+\gamma+\mu+\nu+\rho) \delta^{(2)} \mathcal{R} \delta^{(2)} \mathcal{R} \delta^{(2)} \mathcal{R} \delta^{(2)} \mathcal{R}
$$

Expanding Eq. (27), one finds that

$$
\begin{aligned}
\left\langle h^{4}\right\rangle_{4}= & 4(\alpha+\beta+\gamma+\mu+\nu+\rho) \sqrt{-g}\left(g^{x x} g^{y y}\right)^{2}\left[\left(\omega_{1} g^{t t} \omega_{2}+k_{1} g^{z z} k_{2}\right)\left(\omega_{2} g^{t t} \omega_{3}+k_{2} g^{z z} k_{3}\right)\right. \\
& \left.\times\left(\omega_{3} g^{t t} \omega_{4}+k_{3} g^{z z} k_{4}\right)\left(\omega_{1} g^{t t} \omega_{4}+k_{1} g^{z z} k_{4}\right)\right] h_{x y}^{(1)} h_{x y}^{(2)} h_{x y}^{(3)} h_{x y}^{(4)},
\end{aligned}
$$

which can then be used to construct a $2 n$-point amplitude for any $n \geq 2$,

$$
\begin{aligned}
\left\langle h^{2 n}\right\rangle_{4}= & 4(\alpha+\beta+\gamma+\mu+\nu+\rho)\left(\begin{array}{c}
2 n \\
4
\end{array}\right) \sum_{p=0}^{n-2}\left(\begin{array}{c}
n+1-p \\
3
\end{array}\right) \Theta(p) \sqrt{-g}\left(g^{x x} g^{y y}\right)^{2} \\
& \times\left[\left(\omega_{1} g^{t t} \omega_{2}+k_{1} g^{z z} k_{2}\right)\left(\omega_{2} g^{t t} \omega_{3}+k_{2} g^{z z} k_{3}\right)\left(\omega_{3} g^{t t} \omega_{4}+k_{3} g^{z z} k_{4}\right)\left(\omega_{1} g^{t t} \omega_{4}+k_{1} g^{z z} k_{4}\right)\right] \\
& \times h_{x y}^{(1)} h_{x y}^{(2)} h_{x y}^{(3)} h_{x y}^{(4)} \prod_{j=3}^{n}\left[\left(h_{y}^{x}\right)^{(2 j-1)}\left(h_{x}^{y}\right)^{(2 j)}\right],
\end{aligned}
$$

where the combinatoric factors (here and below) are handled similarly to those in Eq. (23).

As for the basis 6-point amplitude, this goes as

$$
\begin{aligned}
\left\langle h^{6}\right\rangle_{6}= & (4 \rho+6(\alpha+\beta+\gamma+\mu+\nu)) \sqrt{-g}\left(g^{x x} g^{y y}\right)^{3} \\
& \times\left[\left(\omega_{1} g^{t t} \omega_{2}+k_{1} g^{z z} k_{2}\right)^{2}\left(\omega_{3} g^{t t} \omega_{4}+k_{3} g^{z z} k_{4}\right)\left(\omega_{5} g^{t t} \omega_{6}+k_{5} g^{z z} k_{6}\right)\right] \\
& \times h_{x y}^{(1)} h_{x y}^{(2)} h_{x y}^{(3)} h_{x y}^{(4)} h_{x y}^{(5)} h_{x y}^{(6)},
\end{aligned}
$$

from which one can construct a $2 n$-point amplitude for any $n \geq 3$,

$$
\begin{aligned}
\left\langle h^{2 n}\right\rangle_{6}= & (4 \rho+6(\alpha+\beta+\gamma+\mu+\nu))\left(\begin{array}{c}
2 n \\
6
\end{array}\right) \sum_{q=0}^{n-3}\left(\begin{array}{c}
n-q+2 \\
5
\end{array}\right) \Theta(q) \sqrt{-g}\left(g^{x x} g^{y y}\right)^{3} \\
& \times\left[\left(\omega_{1} g^{t t} \omega_{2}+k_{1} g^{z z} k_{2}\right)^{2}\left(\omega_{3} g^{t t} \omega_{4}+k_{3} g^{z z} k_{4}\right)\left(\omega_{5} g^{t t} \omega_{6}+k_{5} g^{z z} k_{6}\right)\right] \\
& \times h_{x y}^{(1)} h_{x y}^{(2)} h_{x y}^{(3)} h_{x y}^{(4)} h_{x y}^{(5)} h_{x y}^{(6)} \prod_{k=4}^{n}\left[\left(h_{y}^{x}\right)^{(2 k-1)}\left(h_{x}^{y}\right)^{(2 k)}\right] .
\end{aligned}
$$

Finally, the basis 8-point amplitude is of the form

$$
\begin{aligned}
\left\langle h^{8}\right\rangle_{8}= & \left(\rho+\frac{1}{2}(\alpha+\beta+\gamma+\mu+\nu)\right)\left(g^{x x} g^{y y}\right)^{4}\left(\omega_{1} g^{t t} \omega_{2}+k_{1} g^{z z} k_{2}\right)\left(\omega_{3} g^{t t} \omega_{4}+k_{3} g^{z z} k_{4}\right) \\
& \times\left(\omega_{5} g^{t t} \omega_{6}+k_{5} g^{z z} k_{6}\right)\left(\omega_{7} g^{t t} \omega_{8}+k_{7} g^{z z} k_{8}\right) h_{x y}^{(1)} h_{x y}^{(2)} h_{x y}^{(3)} h_{x y}^{(4)} h_{x y}^{(5)} h_{x y}^{(6)} h_{x y}^{(7)} h_{x y}^{(8)},
\end{aligned}
$$

and the corresponding $2 n$-point amplitude for any $n \geq 4$ is then

$$
\begin{aligned}
\left\langle h^{2 n}\right\rangle_{8}= & \left(\rho+\frac{1}{2}(\alpha+\beta+\gamma+\mu+\nu)\right)\left(\begin{array}{c}
2 n \\
8
\end{array}\right) \sum_{r=0}^{n-4}\left(\begin{array}{c}
n-r+3 \\
7
\end{array}\right) \Theta(r) \sqrt{-g}\left(g^{x x} g^{y y}\right)^{4} \\
& \times\left(\omega_{1} g^{t t} \omega_{2}+k_{1} g^{z z} k_{2}\right)\left(\omega_{3} g^{t t} \omega_{4}+k_{3} g^{z z} k_{4}\right)\left(\omega_{5} g^{t t} \omega_{6}+k_{5} g^{z z} k_{6}\right)\left(\omega_{7} g^{t t} \omega_{8}+k_{7} g^{z z} k_{8}\right) \\
& \times h_{x y}^{(1)} h_{x y}^{(2)} h_{x y}^{(3)} h_{x y}^{(4)} h_{x y}^{(5)} h_{x y}^{(6)} h_{x y}^{(7)} h_{x y}^{(8)} \prod_{l=5}^{n}\left[\left(h_{y}^{x}\right)^{(2 l-1)}\left(h_{x}^{y}\right)^{(2 l)}\right] .
\end{aligned}
$$

As before, the complete $2 n$-point amplitude for the Riem ${ }^{4}$ theory in the high-momentum regime is a linear combination of the basis amplitudes in Eqs. (31), (33), and (35). That is,

$$
\left\langle h^{2 n}\right\rangle_{\operatorname{Riem}^{4}}=\left\langle h^{2 n}\right\rangle_{4}+\left\langle h^{2 n}\right\rangle_{6}+\left\langle h^{2 n}\right\rangle_{8} .
$$




\section{MULTIPOINT AMPLITUDES FROM Riem $q$ $\left(\epsilon^{q-1}\right.$-ORDER) GRAVITY}

In this section, we will find the basic form of the $2 n$-point amplitude in the high-momentum regime when the most general type of gravitational theory is considered, namely, one whose Lagrangian is composed of $q$ contracted Riemann tensors for arbitrary $q$.

This task appears to be quite arduous, as one would expect that the number of gauge-invariant terms in the Lagrangian grows exponentially with $q .^{7}$ On the other hand, as shown in Sec. II C, each of the six invariants makes essentially the same contribution to any one of the three basis amplitudes [cf. Eqs. (30), (32), and (34)]—although the different basis amplitudes will indeed have different forms. It is not difficult to convince oneself that the relative simplicity of the high-momentum regime is enough to ensure that these trends will persist to higher orders in $q$.

Since any single graviton can carry zero, one, or two derivatives, there are many ways to obtain a $2 n$-point amplitude from a $\operatorname{Riem}^{q}$ theory depending on the size and parity of $q$. Let us suppose that this is a $q$-odd theory; then the set of basis amplitudes (i.e., those with only differentiated gravitons) is the set $P_{q}=\left\{\left\langle h^{q+1}\right\rangle_{q+1},\left\langle h^{q+3}\right\rangle_{q+3}, \ldots,\left\langle h^{2 q}\right\rangle_{2 q}\right\}$ with cardinality $\frac{q+1}{2}$. The reasoning here is that, for an odd value of $q,\left\langle h^{q+1}\right\rangle_{q+1}$ has the maximum possible number of gravitons carrying two derivatives with the remainder carrying one, whereas $\left\langle h^{2 q}\right\rangle_{2 q}$ has all gravitons carrying a single derivative.

To elaborate further, let us consider the following arrangements of $q$ contracted Riemann tensors [while keeping in mind Eqs. (13) and (14) and that the gravitons come in pairs]:

$$
\begin{gathered}
\left\langle h^{q+1}\right\rangle_{q+1} \sim \underbrace{\sim \delta^{(1)} \mathcal{R} \delta^{(1)} \mathcal{R} \cdots \delta^{(1)} \mathcal{R} \delta^{(2)} \mathcal{R}}_{q \text { products of Riemann tensors }} \text { a single } \delta^{(2)} \mathcal{R} \text { term and an even number of } \delta^{(1)} \mathcal{R} \text { terms, } \\
\left\langle h^{q+3}\right\rangle_{q+3} \sim \underbrace{\delta^{(1)} \mathcal{R} \delta^{(1)} \mathcal{R} \cdots \delta^{(2)} \mathcal{R} \delta^{(2)} \mathcal{R} \delta^{(2)} \mathcal{R}}_{q \text { products of Riemann tensors }} \text { three } \delta^{(2)} \mathcal{R} \text { terms and an even number of } \delta^{(1)} \mathcal{R} \text { terms, } \\
\vdots \\
\vdots \quad \vdots \\
\left\langle h^{2 q}\right\rangle_{2 q} \sim \underbrace{\delta_{(2)} \mathcal{R} \delta^{(2)} \mathcal{R} \cdots \delta^{(2)} \mathcal{R} \delta^{(2)} \mathcal{R}}_{q \text { products of Riemann tensors }}
\end{gathered}
$$

A similar argument can be used for $q$-even theories, in which case, the set of basis amplitudes is $Q_{q}=$ $\left\{\left\langle h^{q}\right\rangle_{q},\left\langle h^{q+2}\right\rangle_{q+2}, \ldots,\left\langle h^{2 q}\right\rangle_{2 q}\right\}$ with cardinality $\frac{q+2}{2}$. In both cases, the key point is that there should be either zero or an even number of $\delta^{(1)} \mathcal{R}$ 's in any basis amplitude.

The next step involves reformulating the different basis amplitudes in terms of gravitons rather than Riemann tensors. It is clear that each such basis amplitude will be a polynomial in $\omega$ 's and $k$ 's of degree $2 q$. Since either one or two derivatives can act on a graviton, each term in the polynomial must contain one of $\omega_{i}, k_{i}, \omega_{i} k_{i}, \omega_{i}^{2}, k_{i}^{2}$ for each graviton $h_{x y}^{(i)}$. Then as long as $q \leq 2 p<2 q$ for some $p \in \mathbb{N}^{+}$, a basis amplitude for Riem ${ }^{q}$ can be expressed somewhat schematically as ${ }^{8}$

$$
\left\langle h^{2 p}\right\rangle_{2 p}=\mathcal{A}_{q} 2^{q+2-2 p} \sqrt{-g}\left(g^{x x} g^{y y}\right)^{p} \underbrace{C_{(1,2)} C_{(3,4)} \cdots C_{(2 p-1,2 p)} C_{(1,2 p)} \cdots}_{q \text { contractions of derivatives }} h_{x y}^{(1)} h_{x y}^{(2)} h_{x y}^{(3)} h_{x y}^{(4)} \cdots h_{x y}^{(2 p-1)} h_{x y}^{(2 p)},
$$

where $C_{(i, j)} \equiv K_{i} \cdot K_{j}=\left(\omega_{i} g^{t t} \omega_{j}+k_{i} g^{z z} k_{j}\right)$ with $K_{i}=\left(\omega_{i}, 0,0,0, k_{i}\right)$ and $\mathcal{A}_{q} \sim \mathcal{O}\left(e^{q}\right)$ is a numerical coefficient. For $2 p=2 q$, the basis amplitude should rather be written as

$$
\left\langle h^{2 q}\right\rangle_{2 p}=\mathcal{A}_{q} 2^{2-q} \sqrt{-g}\left(g^{x x} g^{y y}\right)^{q} \underbrace{C_{(1,2)} C_{(3,4)} \cdots C_{(2 q-1,2 q)}}_{q \text { contractions of derivatives }} h_{x y}^{(1)} h_{x y}^{(2)} h_{x y}^{(3)} h_{x y}^{(4)} \cdots h_{x y}^{(2 q-1)} h_{x y}^{(2 q)} .
$$

\footnotetext{
${ }^{7}$ It is amusing to note that the number of gauge-invariant terms grows exactly as $(q-1)$ ! for $q \leq 4$, which then grows roughly as $e^{q}$ for large $q$.

${ }^{8}$ This form assumes that the gravitons can be freely labeled.
} 
Now each basis amplitude $\left\langle h^{2 p}\right\rangle_{2 p}$-for which $q \leq 2 p \leq 2 q$ if $q$ is even or $q+1 \leq 2 p \leq 2 q$ if $q$ is odd-will contribute to the $2 n$-point amplitude in accordance with

$$
\begin{aligned}
\left\langle h^{2 n}\right\rangle_{2 p}= & \mathcal{A}_{q} 2^{q+2-2 p}\left(\begin{array}{c}
2 n \\
2 p
\end{array}\right) \sum_{r=0}^{n-p}\left(\begin{array}{c}
n+p-r+1 \\
2 p-1
\end{array}\right) \Theta(r) \sqrt{-g}\left(g^{x x} g^{y y}\right)^{p} \underbrace{C_{(1,2)} C_{(3,4)} \cdots C_{(2 p-1,2 p)} C_{(1,2 p)} \cdots}_{q \text { contractions }} \\
& \times h_{x y}^{(1)} h_{x y}^{(2)} \cdots h_{x y}^{(2 p-1)} h_{x y}^{(2 p)} \prod_{m=p+1}^{n}\left[\left(h_{y}^{x}\right)^{(2 m-1)}\left(h_{x}^{y}\right)^{(2 m)}\right],
\end{aligned}
$$

for all $n \geq p$, and the combinatoric factors follow the same logic as in the analysis from Sec. II.

Finally, we can use Eq. (42) to express the complete $2 n$-point for any $\operatorname{Riem}^{q}$ theory gravity as the sum of contributions from the various basis amplitudes,

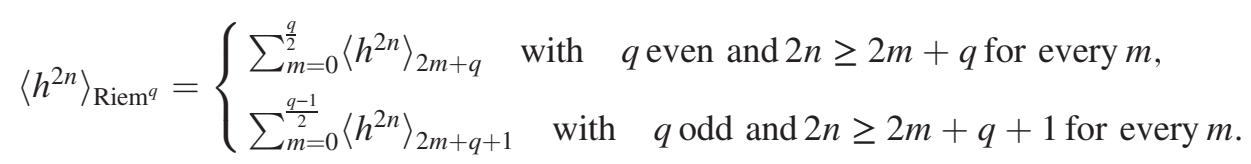

It should again be emphasized that the validity of these results depends upon the restriction to the high-momentum regime.

\section{SCATTERING PROPERTIES OF 2n-POINT AMPLITUDES}

Our next order of business is to look at the scattering properties of some of these amplitudes. We will restrict to the cases with four gravitons (all of which are differentiated) as then the results can be expressed directly in terms of the familiar Mandelstam variables [see Eqs. (46) to (48) below]. However, it can be expected that the same basic theme-each theory carrying its own characteristic signature for scattering experiments in the high-momentum regime-will persist to more complicated scenarios.

Let us begin with the 4-point amplitude of Riem ${ }^{3}$ gravity as depicted in Sec. II B. It is convenient to express the products of momenta in terms of the condensed notation $K_{i} \cdot K_{j}=\left(\omega_{i} g^{t t} \omega_{j}+k_{i} g^{z z} k_{j}\right)$, in which case,

$$
\left\langle h^{4}\right\rangle=-6(\alpha+\beta) \sqrt{-g}\left(g^{x x} g^{y y}\right)^{2} K_{(1} \cdot K_{2} K_{3} \cdot K_{4} K_{1} \cdot K_{4} h_{x y}^{(1)} h_{x y}^{(2)} h_{x y}^{(3)} h_{x y}^{(4)} .
$$

It should be noted that the symmetrized product of momenta in Eq. (44) is really a shorthand for the symmetrization of all possible products in a particular way,

$$
\begin{aligned}
K_{(1} \cdot K_{2} K_{3} \cdot K_{4} K_{1} \cdot K_{4)} \rightarrow & K_{(1} \cdot K_{2} K_{3} \cdot K_{4} K_{1} \cdot K_{4)}+K_{(1} \cdot K_{2} K_{3} \cdot K_{4} K_{1} \cdot K_{2)}+K_{(1} \cdot K_{2} K_{3} \cdot K_{4} K_{1} \cdot K_{3)} \\
& +K_{(1} \cdot K_{2} K_{3} \cdot K_{4} K_{2} \cdot K_{3)}+K_{(1} \cdot K_{2} K_{3} \cdot K_{4} K_{2} \cdot K_{4)}+K_{(1} \cdot K_{2} K_{3} \cdot K_{4} K_{3} \cdot K_{4)} .
\end{aligned}
$$

To better appreciate Eq. (45), one can take note that each of the momenta has an equal opportunity of appearing either once or twice in any given permutation of the four gravitons.

Let us now recall the Mandelstam variables,

$$
\begin{gathered}
s=\left(K_{1}+K_{2}\right)^{2}=\left(K_{3}+K_{4}\right)^{2}=2 K_{1} \cdot K_{2}=2 K_{3} \cdot K_{4}, \\
t=\left(K_{1}-K_{3}\right)^{2}=\left(K_{2}-K_{4}\right)^{2}=-2 K_{1} \cdot K_{3}=-2 K_{2} \cdot K_{4}, \\
u=\left(K_{1}-K_{4}\right)^{2}=\left(K_{2}-K_{3}\right)^{2}=-2 K_{1} \cdot K_{4}=-2 K_{2} \cdot K_{3},
\end{gathered}
$$


where the two rightmost pairs of expressions on each line represents the case of massless particles, which is the case of current interest and for which $s+t+u=0$. In terms of its dependency on the Mandelstam variables, Eq. (44) can be expressed as

$$
\left\langle h^{4}\right\rangle_{\operatorname{Riem}^{3}} \propto(s-t-u)\left(s^{2}+t^{2}+u^{2}\right) h_{x y}^{(1)} h_{x y}^{(2)} h_{x y}^{(3)} h_{x y}^{(4)} .
$$

In the interest of making a connection with the gauge theory, it should be emphasized that Eq. (49) is only valid at the AdS boundary where $g_{z z}=\left|g_{t t}\right|$ holds true. But, since the idea is to translate these expressions into statements in the dual gauge theory (as in [13]), the boundary limit is sufficient. Indeed, we expect to observe a related signature for the stress-energy correlators in the gauge theory. This is because of the observation in [9] that the amplitudes which survive in the high-momentum regime are mostly unaffected by the process of holographic renormalization.

We can similarly apply this process to the 4-point amplitude from Riem ${ }^{4}$ gravity, which leads to

$$
\begin{aligned}
\left\langle h^{4}\right\rangle_{\operatorname{Riem}^{4}} \propto & \left(s^{4}+t^{4}+u^{4}+s^{2} t^{2}+s^{2} u^{2}+u^{2} t^{2}\right) \\
& \times h_{x y}^{(1)} h_{x y}^{(2)} h_{x y}^{(3)} h_{x y}^{(4)}
\end{aligned}
$$

In view of Eqs. (49) and (50), two conclusions immediately follow: The first is that the two theories have very distinct scattering signatures, and the second is that $s, t$, and $u$ appear democratically in both cases. The latter is a consequence of the high-momentum regime favoring no particular scattering channel, meaning that, in general, this is also a regime of large-angle scattering.

\section{CONCLUSION}

In this paper, we have computed graviton multipoint scattering amplitudes for higher-derivative theories in an
AdS black brane background. All computations were carried out in the so-called high-momentum regime as was first introduced in [9]. This regime allows for higher-curvature corrections to contribute significantly to higher-point amplitudes provided that $s \epsilon \lesssim 1$, where $\epsilon$ is the perturbative ( $\alpha^{\prime}$ or Regge slope) expansion parameter. Along with explicit calculations for six- and eight-derivative theories, we were able to generalize the formalism to higher-derivative gravity of arbitrary order. A critical element of this generalization was the construction of a certain class of basis amplitudes.

We proceeded to demonstrate the scattering properties of Riem ${ }^{3}$ and Riem ${ }^{4}$ gravity in terms of the Mandelstam variables $s$, $t$, and $u$ by using their respective 4-point amplitudes. Our expectation is that this procedure can be generalized to higher-point scattering amplitudes and higher-derivative theories with some amount of work.

The graviton multipoint amplitudes in this paper should correspond to stress-tensor correlators in the gauge theory. This means, for instance, that the stress-energy tensor 4-point correlators should include, in addition to the distinct signatures of Einstein gravity and four-derivative gravity $[9,13]$, those of six and eight-derivative gravity as depicted in Eqs. (49) and (50). Note, however, that, to make contact with actual experiments, it is the connected functions in the gauge theory that are required, whereas the amplitudes in this paper would correspond to one-particle-irreducible functions. This point is currently under investigation by the current authors.

\section{ACKNOWLEDGMENTS}

The research of A. J. M. M. received support from NRF Incentive Funding Grant No. 85353 and NRF Competitive Programme Grant No. 93595. M. M. W. S. is supported by an NRF bursary through Competitive Programme Grant No. 93595 and a Henderson Scholarship from Rhodes University.
[1] J. M. Maldacena, The large $N$ limit of superconformal field theories and supergravity, Int. J. Theor. Phys. 38, 1113 (1999); Advances in Theoretical and Mathematical Physics, Adv. Theor. Math. Phys. 2, 231 (1998).

[2] D. M. Hofman and J. Maldacena, Conformal collider physics: Energy and charge correlations, J. High Energy Phys. 05 (2008) 012.

[3] E. Witten, Anti-de Sitter space and holography, Adv. Theor. Math. Phys. 2, 253 (1998).

[4] O. Aharony, S. S. Gubser, J. M. Maldacena, H. Ooguri, and Y. Oz, Large $N$ field theories, string theory and gravity, Phys. Rep. 323, 183 (2000).

[5] C. Cheung and G. N. Remmen, Positivity of Curvature-Squared Corrections in Gravity, Phys. Rev. Lett. 118, 051601 (2017).
[6] S. Deser, One-loop gravity divergences in $D>4$ cannot all be removed, Gen. Relativ. Gravit. 48, 157 (2016).

[7] G. 't Hooft, An algorithm for the poles at dimension four in the dimensional regularization procedure, Nucl. Phys. B62, 444 (1973).

[8] M. D. Pollock, On the application of the field-redefinition theorem to the heterotic superstring theory, Eur. Phys. J. Plus 130, 87 (2015).

[9] R. Brustein and A. J. M. Medved, Graviton n-point functions for UV-complete theories in anti-de Sitter space, Phys. Rev. D 85, 084028 (2012).

[10] D. Lovelock, The Einstein tensor and its generalizations, J. Math. Phys. 12, 498 (1971). 
[11] J. Casalderrey-Solana, H. Liu, D. Mateos, K. Rajagopal, and U. A. Wiedemann, Gauge/String Duality, Hot QCD and Heavy Ion Collisions (Cambridge University Press, Cambridge, UK, 2014).

[12] R. Brustein and A. J. M. Medved, Universal stress-tensor correlation functions of strongly coupled conformal fluids, Phys. Lett. B 724, 144 (2013).

[13] R. Brustein and A. J. M. Medved, Graviton multipoint functions at the AdS boundary, Phys. Rev. D 87, 024005 (2013).

[14] K. Skenderis, Lecture notes on holographic renormalization, Classical Quantum Gravity 19, 5849 (2002).
[15] P. K. Kovtun and A. O. Starinets, Quasinormal modes and holography, Phys. Rev. D 72, 086009 (2005).

[16] S. Grozdanov and A. O. Starinets, On the universal identity in second order hydrodynamics, J. High Energy Phys. 03 (2015) 007.

[17] G. Policastro, D. T. Son, and A. O. Starinets, From AdS/ CFT correspondence to hydrodynamics, J. High Energy Phys. 09 (2002) 043.

[18] G. 't Hooft and M. J. G. Veltman, One loop divergencies in the theory of gravitation, Ann. Inst. Henri Poincaré Phys. Theor. A 20, 69 (1974).

[19] M. M. W. Shawa and A. J. M. Medved (to be published). 